\title{
Worst-Case Bounds for Subadditive Geometric Graphs
}

\author{
Marshall Bern * $\quad$ David Eppstein ${ }^{\dagger}$
}

November 10, 1992

\begin{abstract}
We consider graphs such as the minimum spanning tree, minimum Steiner tree, minimum matching, and traveling salesman tour for $n$ points in the $d$-dimensional unit cube. For each of these graphs, we show that the worst-case sum of the $d$ th powers of edge lengths is $O(\log n)$. This is a consequence of a general "gap theorem": for any subadditive geometric graph, either the worst-case sum of edge lengths is $O\left(n^{(d-1) / d}\right)$ and the sum of $d$ th powers is $O(\log n)$, or the sum of edge lengths is $\Omega(n)$. We look more closely at some specific graphs: the worst-case sum of $d$ th powers is $O(1)$ for minimum matching, but $\Omega(\log n)$ for traveling salesman tour, which answers a question of Snyder and Steele.
\end{abstract}

\section{Introduction}

A worst-case, or a priori, bound on a geometric graph is a bound that depends only on the assumption that all vertices lie within a given container. Such a bound does not depend on the specific locations of vertices, nor on any probabilistic assumptions.

Early papers especially considered the worst-case length of an optimal traveling salesman tour for $n$ points in the unit square. The current best upper bound [3] is about $1.392 \sqrt{n}+$ $o(\sqrt{n})$ and the best lower bound is about 1.074 $\sqrt{n}-o(\sqrt{n})$. Steele and Snyder [11] showed that this $\Theta\left(n^{(d-1) / d}\right)$ behavior (in fact, convergence to some constant times $n^{(d-1) / d}$ ) holds in any fixed dimension $d$ for both minimum spanning tree (MST) and traveling salesman tour (TSP). Snyder has proved similar results for the rectilinear minimal Steiner tree $[5,6]$.

More recently, Steele and others have looked at the sum of powers of edge lengths for geometric graphs in the unit cube. There are several reasons to consider power-weighted edges. First, an $O(1)$ bound on the sum of $d$ th powers (as is known for MST [2,9]) implies an $O\left(n^{(d-1) / d}\right)$ bound on the sum of edge lengths, so in some sense these are the harder problems. Second, MST computations typically use squared edge lengths in order to avoid taking square roots. Because the MST lexicographically minimizes the vector of edge lengths, this substitution computes the correct tree. In other computations, squares may be substituted for edge lengths as an inexact heuristic. Finally, in some models of VLSI circuits, delay along wires scales as the square of the edge length, while other measures (e.g., capacitance) scale linearly.

\footnotetext{
${ }^{*}$ Xerox PARC, 3333 Coyote Hill Rd., Palo Alto, CA, 94304

${ }^{\dagger}$ Department of Information and Computer Science, University of California, Irvine, CA 92717. Work done partially while visiting Xerox PARC.
} 
In considering the sum of $d$ th powers, an important point is that the graph is chosen to minimize the sum of edge lengths, not the sum of power-weighted edges. For example, a space-filling curve heuristic [9, 7] gives a suboptimal TSP tour for which the sum of squares is $O(1)$; the interesting question is whether the shortest TSP tour has this property.

Snyder and Steele [7] recently proved that the sum of squares for the shortest planar TSP tour is $O(\log n)$. In this paper, we extend the result of Snyder and Steele to show that $O(\log n)$ holds for any geometric graph in arbitrary fixed dimension, satisfying certain natural conditions. We also give an $O(1)$ bound for minimum matching and an $\Omega(\log n)$ lower bound for TSP. This second result is somewhat surprising, because asymptotic growth rates (both probabilistic and worst-case) are typically identical for different geometric graphs in common dimension. The only previous surprises involve bipartite matchings $[1,4]$; the growth rate for the planar probabilistic case is $\Theta\left((n \log n)^{1 / 2}\right)$, rather than the usual $\Theta\left(n^{1 / 2}\right)[1]$.

\section{Gap Theorem}

A geometric graph is a graph with vertices in Euclidean $d$-space, and edges that are line segments joining pairs of vertices. Edge lengths are given by Euclidean distances between vertices. Let $G$ be a function that maps each finite subset $X$ of the unit cube $[0,1]^{d}$ to a geometric graph $G(X)$ with vertex set $X$. Let $n$ denote $|X|$, and assume that $d$ is fixed. Let $L(G(X))$ denote the sum of the lengths of the edges in $G(X)$ and $P(G(X))$ denote the sum of the $d$ th powers of edge lengths.

The combinatorial optimization problems of interest (MST, TSP, minimum matching) require some tie-breaking rule so that they are well-defined on degenerate point sets. We assume a tie-breaking rule of the form: perturb the point set very slightly to obtain an input in general position, map to the graph, and then perturb back. (The bounds in this paper actually hold for all tie-breaking rules, but this assumption simplifies the discussion.)

We assume the following conditions on function $G$. The third condition is the operative one; it is related to the subadditivity conditions defined by Steele $[8,9,11]$. The essential difference is that previous subadditivity conditions subdivide the unit cube and bound $L(G(X))$ by the sum of $L\left(G\left(X \cap C_{i}\right)\right)$ values on the small cubes $C_{i}$, rather than bounding induced subgraphs of $G(X)$. Steele's definitions of subadditivity provide a recursive inequality that allows limit theorems (that is, statements of the form $L(G(X)) \sim \beta_{d} n^{(d-1) / d}$ ), rather than just order results. On the other hand, our definition is less restrictive and easier to confirm.

1. (Translation) If $X^{\prime}$ is obtained from $X$ by a translation, then $G\left(X^{\prime}\right)$ is the same graph (i.e., identical except for locations of vertices) as $G(X)$.

2. (Scaling) If $X^{\prime}$ is obtained from $X$ by scaling, then $G\left(X^{\prime}\right)$ is the same graph as $G(X)$.

3. (Subadditivity) There is a constant $c$, such that for all $X$ and all $X^{\prime} \subset X$, the sum of the lengths of edges in the subgraph of $G(X)$ induced by $X^{\prime}$ is at most $c \cdot L\left(G\left(X^{\prime}\right)\right)$. In other words, an induced subgraph of $G(X)$ is, up to a constant factor, no longer than the graph given by $G$ on the subgraph's vertices. 
In some circumstances we assume a fourth condition, and at other times it follows from the first three assumptions:

4. (Sparsity) For any $i$, there are $O(n)$ edges in $G(X)$ with lengths in the interval $\left[2^{-i}, 2^{-i+1}\right)$.

Theorem 1. Let $G$ map finite point sets in the unit cube $[0,1]^{d}$ to geometric graphs and satisfy conditions 1-3. Then one of the following holds:

- $G(X)$ is sparse, $L(G(X))$ is $O\left(n^{(d-1) / d}\right)$, and $P(G(X))$ is $O(\log n)$, or

- $L(G(X))$ is $\Omega(n)$, and if $G(X)$ is sparse then $P(G(X))$ is $\Omega(n)$.

Proof: We divide edges into length classes. All edges with lengths in the interval $\left[2^{-i}, 2^{-i+1}\right)$ are in class $i$, for $i=0,1,2, \ldots$. The diameter sphere of an edge $a b$ is the smallest sphere passing through vertices $a$ and $b$.

We first consider the case that there is a constant bound on the number of diameter spheres of $G(X)$ edges from a single length class that can enclose a point of $[0,1]^{d}$. This constant bound must hold over all point sets, and we call the maximum number enclosing a point the overlap. Notice that constant overlap implies that each vertex is incident to only $O(1)$ edges from a single length class, which in turn implies sparsity. Constant overlap also implies that the total volume of all diameter spheres for class $i$ is $O(1)$. For class $i>1+2 \log _{2} n$, an edge has length less than $1 / n^{2}$, so the volume of its diameter sphere is $O\left(n^{-2 d}\right)$. The total volume of these small spheres is $O(1)$. Hence the total volume of all diameter spheres and the sum of $d$ th powers $P(G(X))$ are both $O(\log n)$.

The sum of edge lengths, $L(G(X))$, is at most

$$
\sum_{i=0}^{\infty} 2^{-i+1} \cdot c^{\prime} \min \left\{n, 2^{d i}\right\},
$$

where $c^{\prime}$ is a constant. The number of edges of class $i$ must be $O\left(2^{d i}\right)$ because of constant overlap, and must be $O(n)$ because each vertex is incident to only $O(1)$ class $i$ edges. This sum evaluates to $O\left(n^{(d-1) / d}\right)$.

Now assume the opposite: the number of diameter spheres of edges of length class $i$ enclosing a single point of space can grow without bound. Look at the endpoints $X^{\prime}$ of a set of edges $E^{\prime}$ whose diameter spheres all enclose the same point. Subadditivity implies that $L\left(G\left(X^{\prime}\right)\right)$ is at least a constant times the total length of edges in $E^{\prime}$. The translation and scaling conditions let us magnify the point set $X^{\prime}$ by a factor of $2^{i-2}$ so that it approximately fills the unit cube. In this way we can create a sequence of point sets for which the sum of edge lengths is at least a constant times the cardinality of the point set. If $G(X)$ is sparse and $L(G(X))$ is $\Omega(n)$, then a constant fraction of $L(X)$ must come from the first $O(1)$ length classes, so $P(G(X))$ will also be $\Omega(n)$.

Corollary 1. The worst-case sum of the $d$ th powers of edge lengths is $O(\log n)$, and the worst-case sum of edge lengths is $\Theta\left(n^{(d-1) / d}\right)$, for the following geometric graphs: minimum spanning tree, minimum-length matching, TSP tour, $k$-nearest neighbor graph (in which each vertex has an edge to its $k$ nearest neighbors), minimum-length $k$-edge-connected graph, and minimum-length $k$-vertex-connected graph. In each case, the graph may be defined using any $L_{p}$ metric, $1 \leq p \leq \infty$, in place of the usual Euclidean distance. 
Proof: These graphs are clearly invariant under scaling and translation. First, we show that each graph is subadditive. If $G$ is the MST or the minimum matching, the induced subgraph must be a subgraph of $G\left(X^{\prime}\right)$. For the TSP tour, the subgraph induced by $X^{\prime}$ is a TSP tour in a metric in which we introduce shortcuts. That is, we place zero distance between each pair of vertices in $X^{\prime}$ that are the endpoints of a path in $G(X)$ outside the subgraph; these shortcuts can only reduce the length from the true TSP tour $G\left(X^{\prime}\right)$, so subadditivity holds with $c=1$. For $k$-connected graphs and $k$-nearest-neighbor graphs, the subgraph induced by any set $X^{\prime}$ can be replaced by $G\left(X^{\prime}\right)$ and the appropriate connectivity properties remain true, so we can again take $c=1$.

To show that each of these graphs lies on the low side of the gap, we extrapolate from the worst-case bound for the TSP tour. Let $G_{k}(X)$ be the graph formed by joining two points if they are within $k$ edges of each other in this tour. Then $L\left(G_{k}(X)\right)$ is $O\left(k^{2} \ell(X)\right)=O(\ell(X))$, where $\ell(X)$ is the length of the TSP tour. $G_{k}(X)$ is $k$-connected and contains at least $k$ neighbors of each point. $G_{1}(X)$ contains both a matching and a spanning tree. So in all cases $L\left(G_{k}(X)\right)$ is $O(\ell(X))$.

For each of the graphs, a regular grid of points in the unit cube shows that $L(G(X))$ is $\Omega\left(n^{(d-1) / d}\right)$.

There are many graphs satisfying our hypotheses on the other side of the gap, that is, ones for which $L(G(X))=\Omega(n)$. Examples include the complete graph, the maximum spanning tree, and the maximum matching. A more interesting example is the Delaunay triangulation: it is subadditive, since the induced subgraph of $X^{\prime}$ is a subgraph of $G\left(X^{\prime}\right)$, but its length may be as large as $\sqrt{d} n-O(1)$. An optimal graph that fails subadditivity is the minimum-weight star.

\section{Steiner graphs}

We now consider Steiner graphs, those formed by adding extra vertices as well as edges to the original point set. Because there are an infinite number of possible Steiner points, a combinatorial optimization problem does not immediately translate into a function mapping point sets to graphs. For example, it is unknown whether the minimum-weight Steiner triangulation always exists, as there may be a point set with a sequence of more and more complicated triangulations of ever decreasing length. On the other hand, the existence of a minimum has been established for the Steiner tree with any $L_{p}$ metric [2]. For the rest of this section, assume that $G$ represents a well-defined function from point sets to graphs with Steiner points.

The proof of Theorem 1 shows that if $G$ satisfies subadditivity, scaling, and translation, then either $L(G(X))$ is $\Omega(n)$ or the diameter spheres for any given edge length class can have at most $O(1)$ overlap. The latter condition, however, does not immediately give us a worst-case bound as there may be more than $O(n)$ edges per length class. Nevertheless, if we already have a worst-case bound on $L(G(X))$, we can obtain a bound on $P(G(X))$. Alternatively, if we add the assumption of sparsity, the gap theorem then follows.

Theorem 2. Let $G$ be a Steiner graph function satisfying conditions 1-3 and the a priori bound that $L(G(X))$ is $o(n)$. Then $P(G(X))$ is $O(\log n)$. 
Proof: The number of edges in $\left[2^{-i}, 2^{-i+1}\right)$ must be $O\left(2^{d i}\right)$ by the bound on overlap and $o\left(n 2^{i}\right)$ by the a priori bound. So $P(G(X)) \leq \sum_{i=0}^{\infty} 2^{-d i+d} \cdot c^{\prime} \min \left\{2^{d i}, n 2^{i}\right\}$, for some $c^{\prime}$, which again gives $O(\log n)$.

Theorem 3. Let $G$ map finite point sets to geometric graphs in the unit cube $[0,1]^{d}$ and satisfy conditions 1-4. Then one of the following holds:

- $L(G(X))$ is $O\left(n^{(d-1) / d}\right)$, and $P(G(X))$ is $O(\log n)$, or

- $L(G(X))$ is $\Omega(n)$ and $P(G(X))$ is $\Omega(n)$.

Proof: As before, if an unbounded number of diameter spheres can enclose a single point, then $L(G(X))$ and $P(G(X))$ are both $\Omega(n)$. Otherwise, $L(G(X)) \leq \sum_{i=0}^{\infty} 2^{-i+1}$. $\left.c^{\prime} \min \left\{n, 2^{d i}\right\}\right)$ and $\left.P(G(X)) \leq \sum_{i=0}^{\infty} 2^{-d i+d} \cdot c^{\prime} \min \left\{n, 2^{d i}\right\}\right)$ for some $c^{\prime}$, which gives the theorem.

The next result can be obtained from the observation that a mininum Steiner tree is the MST of some point set; our alternative proof illustrates the use of the gap theorem.

Corollary 2. $L(G(X))$ is $\Theta\left(n^{(d-1) / d}\right)$ and $P(G(X))$ is $O(\log n)$ for the minimum Steiner tree of a point set in $d$ dimensions.

Proof: The minimum Steiner tree is subadditive, since any connected component of an induced subgraph is itself a minimum Steiner tree. The Steiner tree satisfies sparsity, since it has $O(n)$ edges overall. And it falls on the small side of the gap, since the total edge length is at most that of the minimum spanning tree. Again a regular grid provides an obvious lower bound example.

\section{Matching}

Corollaries 1 and 2 leave open the question of whether $O(\log n)$ is a tight bound on the sum of $d$ th powers of edge lengths.

Gilbert and Pollak [2] proved that the sum of squares for the planar MST (and hence for minimum Steiner tree) is not just $O(\log n)$, but actually $O(1)$. Specifically, they showed that the edges of an MST have disjoint diamonds, where an edge's diamond is the $60^{\circ}-120^{\circ}$ rhombus with long diagonal equal to the edge. This gives an explicit upper bound of about 5.47. (For MST the best lower bound we know is 3.) The $O(1)$ bound for MST and Steiner tree generalizes to $d$ dimensions; this can be proved either by generalizing diamonds [2] or, more simply, with a space-filling curve argument [9].

In this section, we show that $O(1)$ also holds for minimum matching. We looked at matching because it bears some relationship to the traveling salesman problem. (A TSP tour defines two perfect matchings, and several heuristics for the TSP use minimum matching as a subroutine.) Steele and Snyder [10] have previously shown convergence to constants times $n^{(d-\alpha) / d}$ for the sum of edge lengths to the $\alpha$ power, $0<\alpha<d$, for both worst-case minimum and greedy matchings. 


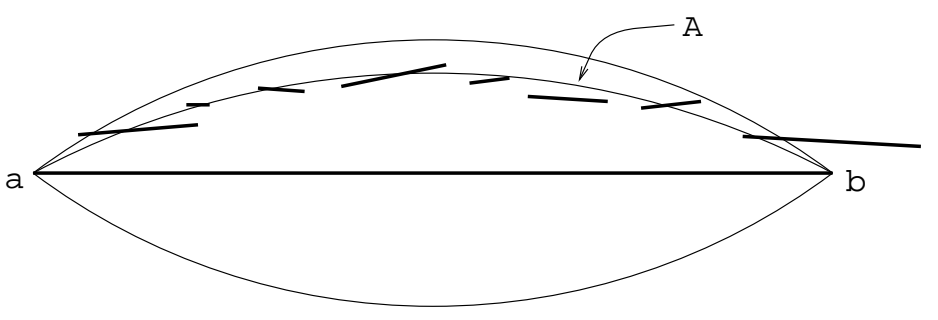

Figure 1. An augmenting cycle within the football of edge $a b$.

Theorem 4. The worst-case sum of the dth powers of edge lengths is $O(1)$ for minimum matching in the unit cube $[0,1]^{d}$.

Proof: We first give the proof in the case $d=2$. We divide edges into $O(1)$ slope classes, and consider only almost-horizontal edges, say with slope in the range $[-.1, .1]$. Let the football of an almost-horizontal edge $a b$ be all points $x$ in the plane such that the angle $a x b$ measures at least $170^{\circ}$. The boundary of a football consists of two circular arcs with endpoints $a$ and $b$, as shown in Figure 1. We shall now show that at most nine-tenths of the area of $a b$ 's football is covered by footballs of edges shorter than $|a b| / 4$. This leaves some constant fraction of $a b$ 's football uncovered; the volume of this fraction is in turn some constant fraction of $|a b|^{2}$.

Aiming for a contradiction, assume nine-tenths of $a b$ 's football is covered by smaller footballs. Then nine-tenths of some circular arc $A$ with endpoints $a$ and $b$ is covered. Centrally project the edges with footballs touching $A$ onto the circle containing $A$. Arbitrarily reduce the resulting collection of arcs so that no more than two arcs cover any point of $A$, and no arc is completely contained in some other arc. Now one or the other way of taking every other arc gives a set of non-overlapping arcs that cover at least four-tenths of $A$. We form a cycle (i.e., a possibly non-simple polygon) by alternating edges and gaps between edges, using $a b$ and the edges corresponding to the selected arcs on $A$. The length of the missing edges in this alternating cycle is less than $1.2|a b|$, because the gaps between arc edges total at most $.8|a b|$, and the gaps between $a$ and the first arc edge and between $b$ and the last arc edge total at most .4 $|a b|$. The length of the edges in the cycle is at least $1.3|a b|$, so the alternating cycle is an augmenting cycle, and the matching cannot be minimum.

We now consider almost-horizontal edges in order of decreasing length. When we come to edge $a b$, we look at its football, which has area proportional to $|a b|^{2}$. No point of $a b$ 's football is covered more than a constant number of times by footballs of edges with length between $|a b|$ and $|a b| / 4$ (or else the worst-case sum of edge lengths would be $\Omega(n)$ by the gap theorem). Moreover, at most nine-tenths of $a b$ 's football is covered by footballs of still smaller edges. So there remains some constant fraction of $a b$ 's football to "pay for" the square of its length. Altogether we can conclude that the sum of squares of almost-horizontal edges is $O(1)$, and hence the sum of squares of all edges is $O(1)$ as well.

The generalization to arbitrary fixed dimension is straightforward. We define the football of $a b$ as above, so that its volume is $\Omega\left(|a b|^{d}\right)$. As above, if $a b$ 's football is nine-tenths covered by smaller footballs, then it must contain a circular arc $A$ that is nine-tenths covered. Constants must be adjusted to establish the augmenting cycle. 

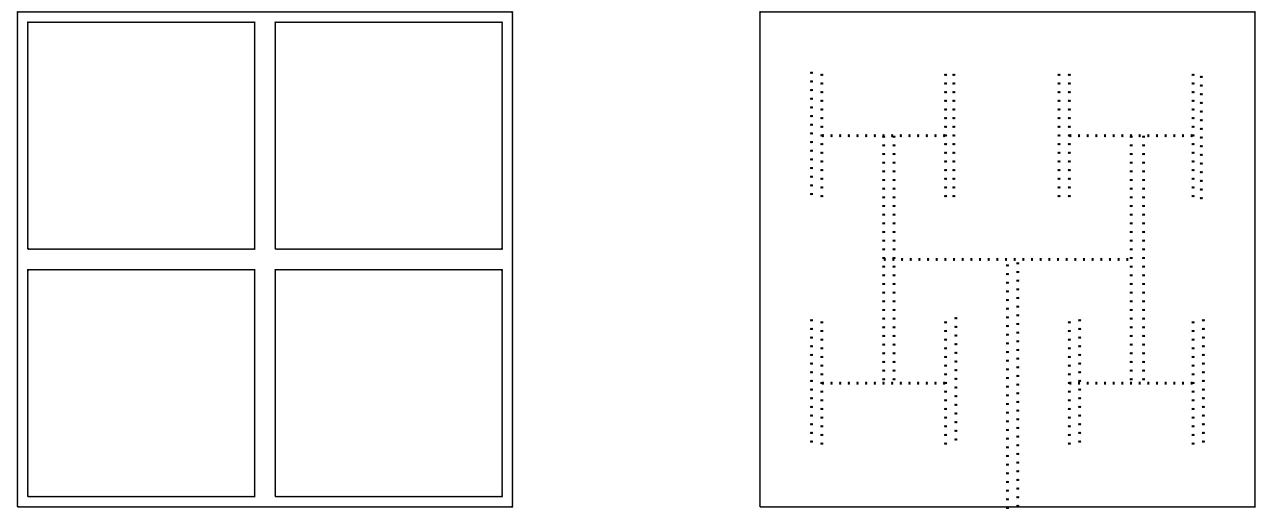

Figure 2. (a) Splitting and shrinking squares. (b) Cities for $k=2$.

\section{Traveling Salesman Tour}

In this section, we answer a question posed by Snyder and Steele [7]. We show that, despite the kinship between minimum matching and the TSP, the sum of $d$ th powers for the TSP may be $\Omega(\log n)$, even in the case $d=2$.

Theorem 5. The worst-case sum of the squares of edge lengths is $\Omega(\log n)$ for the TSP in the unit square.

Proof: We show how to create an infinite sequence of problem instances for which the shortest TSP tour has sum of squares $\Omega(\log n)$. The example is based on the H-tree embedding of a complete binary tree [12].

Let $k$ be a positive integer. We split the unit square into four congruent squares, shrink each of these "child" squares very slightly (by an amount to be determined later), and then split each child. The shrinking leaves vertical and horizontal channels between the child squares; we shall place the points to be toured (the cities) within these channels. We do this splitting and shrinking process $k$ times. See Figure 2(a).

We now describe the placement of the cities. Each time we split a square we place a "T" of cities in the channels. The vertical part of the "T" consists of two parallel lines of cities, with spacing $\epsilon$ between adjacent cities in the same line, and spacing $4 \epsilon$ between lines. The horizontal part of the " $T$ " consists of a single line of cities with spacing $\epsilon$. Two cities are common to both horizontal and vertical lines. See Figure 2(b). The unsplit leaf squares each contain the vertical part of a "T" (just as if they were split), but no horizontal part. Set $\epsilon=2^{-4 k}$ and set the width of all channels to be $8 \epsilon$. This sets the shrinking amount at each level. Lines are centered within channels, so that a city on a vertical line is $2 \epsilon$ away from the closest square boundary.

We now assert that a shortest tour is the obvious one shown in Figure 3(a): simply follow the outside contour of the H-tree. Consider a leaf square $S$ with side length $s$, as shown in Figure 3(b). The intersection of an optimal TSP tour with $S$ is a set of polygonal "loops" with endpoints on the the boundary of $S$. The obvious tour induces a single loop with endpoints $a$ and $b$ and length about $4 s$. Each entry or exit of a loop, except ones within $s / 4$ of the entry and exit of the obvious tour, incurs additional cost of about $s / 4$. 

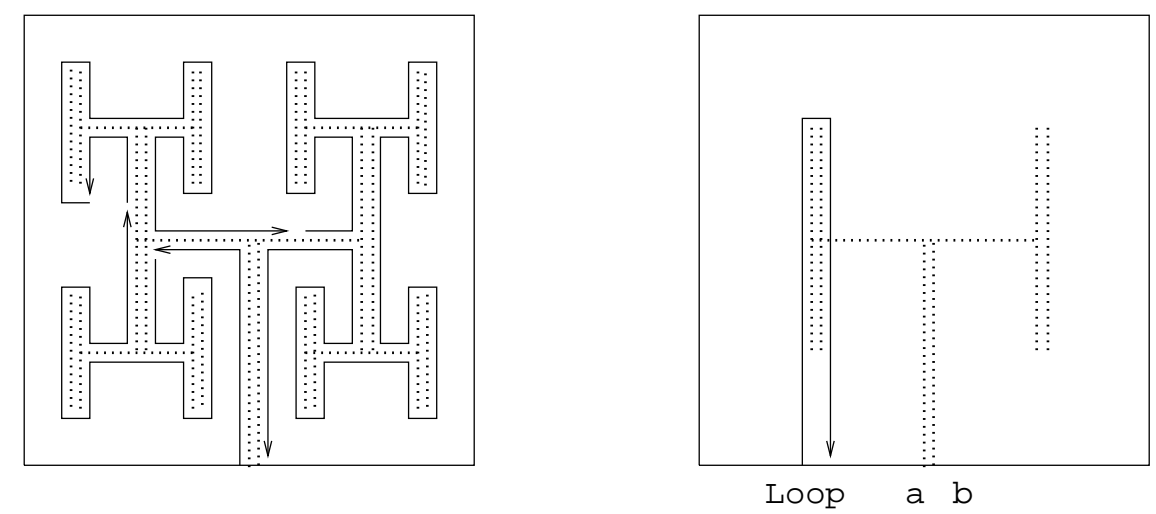

Figure 3. (a) Obvious tour. (b) Within a leaf square.

These additional costs cannot be compensated by savings in a neighboring square, as extra entry and exit points do not help. We can conclude that there is an optimal tour in which $S$ is entered and exited only at $a$ and $b$. We have now reduced to the case of an H-tree with one less level. (The vertical "stem" is included in both the leaf square and the H-tree with one less level, but this does no harm.) The case of $k=1$ is essentially identical to the case of a leaf square.

The total number of cities in our construction is less than $2^{5 k+3}$. In the obvious tour, all edges have length about $\epsilon$ except for the long horizontal edges. The sum of the squares of the long horizontal edges is at least

$$
\frac{1}{2}+4 \cdot\left(\frac{1}{4}-8 \epsilon\right)^{2}+16 \cdot\left(\frac{1}{8}-24 \epsilon\right)^{2}+\ldots+4^{k} \cdot\left(\frac{1}{2^{k}}-8\left(2^{k}-1\right) \epsilon\right)^{2} .
$$

This expression is greater than $k / 8$.

\section{Conclusions}

We have shown that for a large class of natural geometric graphs, the worst-case sum of $d$ th powers of edge lengths is $O(\log n)$ whenever the sum of edge lengths is $O\left(n^{(d-1) / d}\right)$ (in fact, whenever the sum of edge lengths is $o(n))$. This result unifies two previously separate phenomena.

The question of whether $O(\log n)$ is the best possible bound on the sum of $d$ th powers seems to be rather delicate. It would be interesting to give a general theorem that would separate the $O(1)$ geometric graphs (e.g., MST, minimum matching) from the $O(\log n)$ graphs (so far, only TSP). Strengthening our results to convergence results (e.g., sum of $d$ th powers for TSP is asymptotic to $\beta_{d} \log n$ ) would also be worthwhile.

\section{Acknowledgements}

We would like to thank Tim Snyder for some valuable comments. 


\section{References}

[1] M. Ajtai, J. Komlós, and G. Tusnády. On optimal matchings. Combinatorica 4 (1984), $259-264$.

[2] E. N. Gilbert and H. O. Pollak. Steiner minimal trees. SIAM J. Applied Math. 16 (1968), 1-29.

[3] Howard J. Karloff. How long can a Euclidean traveling salesman tour be? SIAM J. Disc. Math. 2 (1989), 91-99.

[4] Peter W. Shor. Random planar matching and bin packing. Ph.D. Thesis, Mass. Inst. of Technology, 1985.

[5] Timothy Law Snyder. Lower bounds for rectilinear Steiner trees in bounded space. Inf. Proc. Letters 37 (1991), 71-74.

[6] Timothy Law Snyder. Worst-case minimal rectilinear Steiner trees in all dimensions. to appear in Disc. and Comp. Geometry.

[7] Timothy Law Snyder and J. Michael Steele. A Priori inequalities for the Euclidean traveling salesman problem. 8th ACM Symp. on Computational Geometry, 1992, 344349 .

[8] J. Michael Steele. Subadditive Euclidean functionals and nonlinear growth in geometric probability. Annals of Probability 9 (1981), 365-376.

[9] J. Michael Steele. Probabilistic and worst case analyses of classical problems of combinatorial optimization in Euclidean space. Math. of Operations Research 15 (1990), 749-770.

[10] J. Michael Steele and Timothy Law Snyder. Worst-case greedy matchings in the unit d-cube. Networks 20 (1990), 779-800.

[11] J. Michael Steele and Timothy Law Snyder. Worst-case growth rates of some classical problems of combinatorial optimization. SIAM J. Computing 18 (1989), 278-287.

[12] Jeffrey D. Ullman. Computational Aspects of VSLI. Computer Science Press, 1984. 